\title{
Status of the LBL Oscillation Experiments MINOS and OPERA
}

\author{
Alfons Weber* \\ for the MINOS and OPERA Collaborations \\ University of Oxford, NAPL, Keble Road, Oxford OX1 3RH, Great Britain \\ E-mail: 'A. Weber1@physics.ox.ac.uki
}

\begin{abstract}
MINOS and OPERA will be the next generation long baseline neutrino experiments to measure neutrino oscillations as suggested by the Super-Kamiokande atmospheric neutrino results in a well controlled environment. Neutrinos produced by the Fermilab main injector will be measured by the two MINOS detectors. A near detector will be located at the Fermilab site to measure the neutrino beam composition at production, a far detector will measure it after oscillation may have modified the beam composition. OPERA will be located in the Grand Sasso underground laboratory and search for the appearance of tau neutrinos in a muon neutrino beam produced at the CERN SPS. By chance, the baseline is $735 \mathrm{~km}$ for both experiments.
\end{abstract}

\section{Introduction}

The most exciting result of the last decade has been the growing evidence that neutrinos

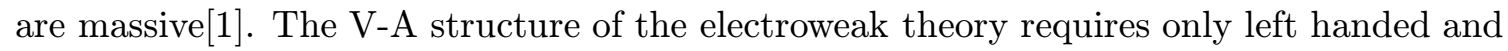
therefore massless neutrinos. Observation of neutrino mass is therefore in direct conflict with the Standard Model.

The most important result, indicating that neutrinos do oscillate and therefore have to have mass, comes from the Super Kamiokande experiment. They observed a zenith angle dependence (and thus a dependence on distance travelled) in the ratio of $\nu_{\mu}$ to $\nu_{e}$ interactions of atmospheric neutrinos [i2]. Since the angular dependence is far larger than the asymmetry in the cosmic ray flux, there is no known way of generating this effect other than neutrino physics. In addition, the shape of the angular dependence is described by oscillation parameters consistent with the measured suppression of the integrated $\nu_{\mu}$ to $\nu_{e}$ ratio. The angular dependence at Super Kamiokande implies that the value of $\Delta \mathrm{m}^{2}$, the difference in the mass squared of the two neutrinos, lies between $10^{-2}$ and $10^{-3} \mathrm{eV}^{2}$. The data from other experiments confirms this observation $\left[\begin{array}{l}\overline{3} \\ \overline{3}\end{array}\right]$.

\footnotetext{
${ }^{*}$ Speaker.
} 


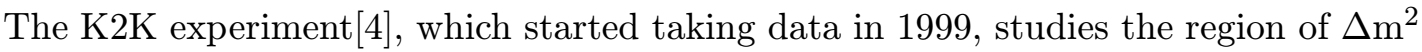
suggested by the atmospheric neutrino experiments. This experiment is sensitive only to some fraction of the region of neutrino mass difference suggested by the atmospheric neutrino results. The beam intensity is not sufficient to cover the whole of the Super-K allowed region and the beam energy is too low to produce $\tau$ leptons in the preferred $\nu_{\mu} \rightarrow \nu_{\tau}$ oscillation mode.

\section{The MINOS Experiment}

The MINOS experiment will study muon neutrinos produced at FNAL in a controlled beam experiment. A beam of 1-20 GeV $\nu_{\mu}$ will be sampled before and after a journey of $735 \mathrm{~km}$ to the Soudan mine in Minnesota. This baseline and choice of energy renders the experiment sensitive to the entire region of neutrino mass difference suggested by the atmospheric neutrino experiments. MINOS will unambiguously confirm or deny the explanation of the atmospheric neutrino anomaly as neutrino oscillations. If neutrinos do oscillate it will provide precise measurements of the $\Delta \mathrm{m}^{2}$ and the mixing matrix elements.

There will be two functionally similar detectors: calorimeters made of planes of 2.54 $\mathrm{cm}$ thick steel interleaved with sensitive planes made up of $1 \mathrm{~cm}$ thick $\mathrm{x} 4 \mathrm{~cm}$ wide solid scintillator strips. The steel plates are octagonal in shape and toroidally magnetised by a coil threaded through a hole in the centre of the octagon. In the far detector the regular octagons are $8 \mathrm{~m}$ in diameter and are grouped into two "supermodules". The total mass of the far detector is 5.4 ktons. The far detector scintillator strips are read out at both ends by wavelength shifting fibres onto Hamamatsu M16 multi-anode phototubes. Eight strips are summed onto each pixel of the phototube. The pattern of summing at each end is arranged such that the strip which is crossed by a single particle is uniquely determined. For multiple hits the event region is well defined. A more detailed description can be found in 阿.

The near detector is offset with respect to the beam in order to prevent the neutrino events from intersecting the coil hole. The squashed octagonal shape of the detector allows the toroidal field to focus muons towards the centre of the detector. Only the inner $25 \mathrm{~cm}$ of the beam will be used in the analysis (the near and far beam spectra are most similar for this region) and so only one quadrant needs to be instrumented in the upstream region. The magnetic field has been designed such that the average field seen by a muon in the near detector is as close as possible to that seen by muons in the far detector. The very large number of neutrino interactions in the near detector opens the possibility of very high statistics measurements of conventional neutrino physics.

The MINOS beam has been designed to use a system of two parabolic horns whose position relative to the target can be adjusted to select a band of neutrino energies. Figure i్di shows the number of $\nu_{\mu}$ CC events per kiloton of detector per year as a function of neutrino energy for three versions of the beam, high, medium and low energy. The beam energy can be chosen to give maximum sensitivity to the region of $\Delta \mathrm{m}^{2}$ to be investigated. It can be seen that beams peaking below $2-3 \mathrm{GeV}$ give essentially negligible event rates, thus setting a lower limit of around $8 \times 10^{-4} \mathrm{eV}^{2}$ on any detectable oscillations. 


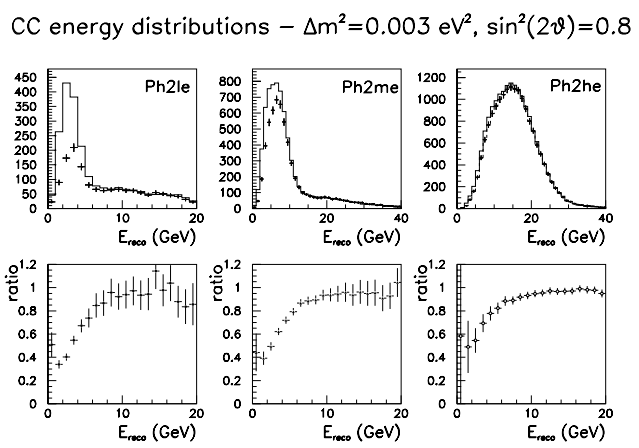

Figure 1: Top: $\nu_{\mu}$ charged current total energy distribution oscillated and unoscillated together for the three different neutrino beam spectra for $\Delta \mathrm{m}^{2}=3 \cdot 10^{-3}$ and $\sin ^{2} 2 \theta=0.8$. Two years of running at nominal intensity have been assumed. Bottom: Ratio of the oscillated and unoscillated energy spectra. A dip can clearly be seen when using the low energy beam. This is the strongest indication for neutrino oscillations.

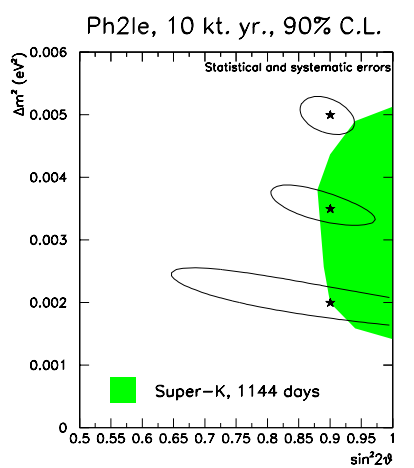

Figure 2: The plot shows the $90 \%$ C.L. allowed regions from fits to reconstructed $\mathrm{CC}$ energy distributions. These distributions are generated with specific oscillation parameters $\Delta \mathrm{m}^{2}, \sin ^{2} 2 \theta$ (indicated by stars on the plot). The Super-K 90\% C.L. allowed region is also shown.

The disappearance of $\nu_{\mu}$ from the beam as a function of energy will be reflected in dips in the measured $\nu_{\mu} \mathrm{CC}$ energy distribution. $\nu_{\mu} \mathrm{CC}$ events can be selected with high purity and small losses by a cut on event length, exploiting the fact that a penetrating muon is present in the final state. Figure ${ }_{1}^{\overline{1}}$ shows the measured CC total energy distribution at Soudan compared with that expected from extrapolation of the near detector energy

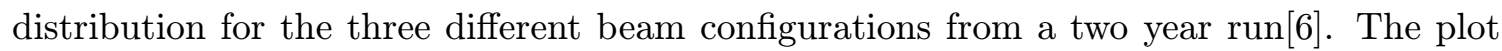
includes all resolution and selection effects. The dips due to the oscillation are most visible with the low energy beam. $\Delta \mathrm{m}^{2}$ is given by the position of the minimum of the dip and $\sin ^{2} 2 \theta$ by the magnitude of the dip. Figure ${ }_{2}^{2}$ shows the confidence level contours in $\Delta \mathrm{m}^{2}-\sin ^{2} 2 \theta$ from fits to distributions generated with $\sin ^{2} 2 \theta=0.8$ and different values of $\Delta \mathrm{m}^{2}$.

Oscillations of $\nu_{\mu} \rightarrow \nu_{\tau}$ or $\nu_{\mu} \rightarrow \nu_{e}$ result in the rate of $\nu_{\mu} \mathrm{CC}$ events being reduced relative to that expected from the near detector rate and being replaced by $\nu_{\tau}$ or $\nu_{e}$ events. $\nu_{\mu} \mathrm{CC}$ events are characterised as "long" events with a penetrating muon whereas $\nu_{\tau}$ and $\nu_{e} \mathrm{CC}$ events, along with neutral current (NC) events, produce only hadronic or electromagnetic showers and are thus characterised as "short" (apart from the $17 \%$ of $\tau$ decays to $\mu \nu \nu)$. The result of oscillations is thus to increase the ratio of short to long events ( $\mathrm{NC} / \mathrm{CC}$ ratio). The measurement of this ratio in the near and far detector is thus a very sensitive indication of oscillations and gives the measurement of oscillation probability with the smallest systematic errors. This and the $\nu_{e}$ - appearance measurement are the two other important measurements done with MINOS. By combining all these measurements it will be possible to explicitly calculate the oscillation probabilities for $\nu_{\mu} \rightarrow \nu_{\tau}, \nu_{\mu} \rightarrow \nu_{e}$ and 
$\nu_{\mu} \rightarrow \nu_{s}$

\section{The OPERA Experiment}

OPERA [i⿱亠凶禸] $]$ is designed as an appearance search for $\nu_{\mu} \rightarrow \nu_{\tau}$ oscillations. It is located in the Gran Sasso Laboratory in the CNGS neutrino beam from the CERN SPS. The detector is based on a massive lead/emulsion target. The nuclear emulsions are exploited for the direct observation of the decay of the $\tau$-lepton, produced in $\nu_{\tau}$ charged current interaction.

While the NuMI beam at Fermilab is optimised to see the dip in the $\nu_{\mu}$ charged current energy distribution, the CNGS beam has been optimised to detect the appearance of $\nu_{\tau}$ events. Its average energy is much higher and thus above the energy threshold for $\tau$ production. The neutrino beam is generated from a $400 \mathrm{GeV}$ proton beam on a graphite target $\left(4.5 \times 10^{19}\right.$ pot/year $)[i \bar{i}$. The pions and kaons produced in this beam are focussed with a magnetic horn and reflector into the $900 \mathrm{~m}$ decay pipe. The expected neutrino spectrum at Grand Sasso can be seen in figure $\underline{\underline{3}}_{r}$. This is predominately a $\nu_{\mu}$ beam with negligible contaminations from electron or $\tau$ neutrinos.

OPERA aims to detect the $\nu_{\tau}$ charged current events by identifying the $\tau$ produced through its decay kink. OPERA is an evolution of the Emulsion Cloud Chamber (ECC) technique, which combines high precision tracking in the emulsion with a passive material to provide the large target mass.

An ECC cell consists of a $1 \mathrm{~mm}$ thick lead plate followed by a thin film made of two $50 \mu \mathrm{m}$ thick emulsion layers separated by a $200 \mu \mathrm{m}$ plastic base. The basic detector unit is obtained by stacking 56 cells to form a compact brick $\left(10.2 \times 12.7 \mathrm{~cm}^{2}\right.$ transverse section, $10 X_{0}$ length, $8.3 \mathrm{~kg}$ weight), which will be assembled into walls.

After each wall, an electronic tracking detector

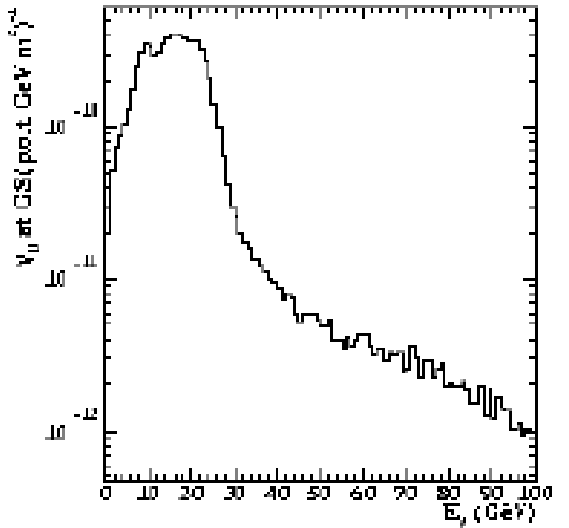

Figure 3: Flux of $\nu_{\mu}$ from CNGS at the OPERA site. is used to select the brick where the neutrino interaction occurs. This tracking detector follows very closely that of MINOS, with scintillator strips coupled to wave-length shifting fibres and multi-anode PMT readout, where the selected PMTs are the same as those used in MINOS near detector. A wall of bricks and two planes of electronic detectors constitute one OPERA module, whose structure is then repeated 24 times to form a supermodule, also including a downstream muon spectrometer. Each muon spectrometer consists of a dipolar magnet (1.55 $\mathrm{T}$ in the tracking region), made of two vertical walls of iron layers, interleaved with RPC detectors and drift tubes, placed in front and behind the magnet as well as between the two walls. Three supermodules form the full OPERA detector, for a total target mass of about $1.8 \mathrm{ktons}$.

The data from the electronic detectors, which will be analysed quasi-online, are used to select bricks where neutrino interactions occur and which will be removed by an automated system. Additional bricks might need to be removed for a complete reconstruction of the 
interesting events. Events selected as $\tau$ candidates will then be sent to dedicated scanning stations, where further studies can be performed to achieve the required background suppression. The expected rate of $\nu_{\mu} \mathrm{CC}$ events is of about 30 events per day.

The $\tau$ decay channels considered by OPERA are listed in Table $\underline{i}_{\mathfrak{r}}^{i}$ together with the corresponding $\nu_{\tau}$ detection efficiencies and the expected number of background events, which are mostly from charm production, large angle muon scattering and hadron reinteractions. New studies are underway to improve the experiment sensitivity by adding also the $\tau \rightarrow \rho \nu$ channel (23.5\% B.R.) to the decay modes considered. The expected number of $\tau$ events per year is given in Table $2 ;$ as a function of possible values of $\Delta \mathrm{m}^{2}$.

\begin{tabular}{|c||c|c|}
\hline Decay mode & $\epsilon\left(\nu_{\tau} C C\right)(\%)$ & $N_{B K G D} /$ year \\
\hline \hline$\tau \rightarrow e$ & 3.7 & 0.04 \\
$\tau \rightarrow \mu$ & 2.7 & 0.03 \\
$\tau \rightarrow h$ & 2.3 & 0.05 \\
\hline
\end{tabular}

\begin{tabular}{|c|c|}
\hline$\Delta \mathrm{m}^{2}\left(\mathrm{eV}^{2}\right)$ & $N_{\tau} /$ year \\
\hline \hline $1.5 \times 10^{-3}$ & 0.82 \\
$2.5 \times 10^{-3}$ & 2.82 \\
$3.2 \times 10^{-3}$ & 3.66 \\
\hline
\end{tabular}

Table 1: OPERA efficiency for the $\tau$ decay channels considered and the corresponding expected number of background events per year. The efficiencies also include the

Table 2: Expected number of $\tau$ events observed in OPERA per year as a function of possible values of $\Delta \mathrm{m}^{2}$ (from ref. $[\overline{\underline{p}} \bar{l})$.

branching ratio for each decay channel.

If no signal is observed, the average upper limit at $90 \%$ C.L. which would be obtained by OPERA is shown in Figure 焦 for 2 and 5 years of exposure respectively. On the other hand, if $\nu_{\tau}$ events are actually observed, a measurement of $\Delta \mathrm{m}^{2}$ can be performed. Assuming maximal mixing and $\Delta \mathrm{m}^{2}=3.2 \times 10^{-3} \mathrm{eV}^{2}$, the $90 \%$ C.L. allowed region for the oscillation parameters as determined by OPERA after 5 years of data taking is shown in Figure $\overline{1}$ :

OPERA also plan to perform a $\nu_{\mu} \rightarrow \nu_{e}$ oscillation analysis, by looking for $\nu_{e}$ appearance above the $\sim 1 \%$ intrinsic $\nu_{e}$ contamination, and to search for neutrino oscillations by studying the ratio between $\mathrm{NC}$ and $\mathrm{CC}$ interactions. However the study of the systematic uncertainties for those analyses is likely to be somewhat limited by the lack of a near detector.

\section{Conclusion}

MINOS and OPERA are two complementary experiments, which will start data taking around 2005. Once and for all they will resolve the atmospheric neutrino problem, measure the parameters of neutrino oscillation, and determine into which type of neutrino $\nu_{\mu} \mathrm{s}$ oscillate.

\section{Acknowledgments}

I would like to thanks the MINOS and OPERA collaborations for providing multiple inputs to this status report. Special thanks to J. Cobb and P. Strolin for carefully reading the talk and this proceedings. 


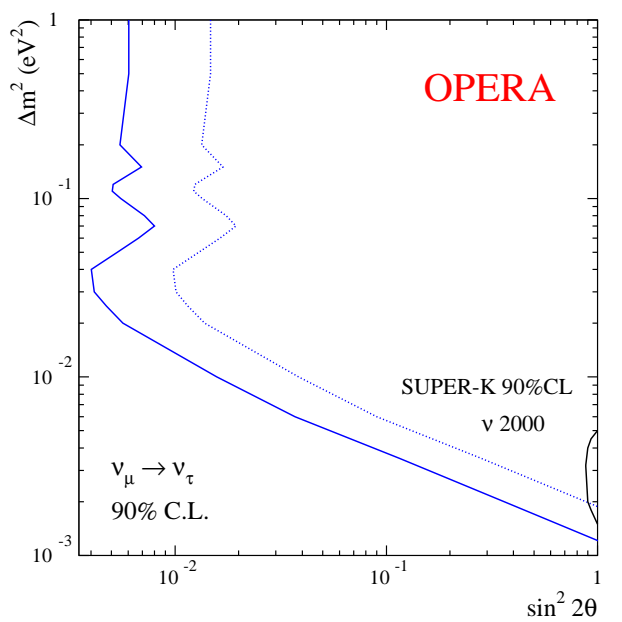

Figure 4: Expected upper limit at the $90 \%$ C.L. from OPERA with no oscillation signal after 2 and 5 years of running.

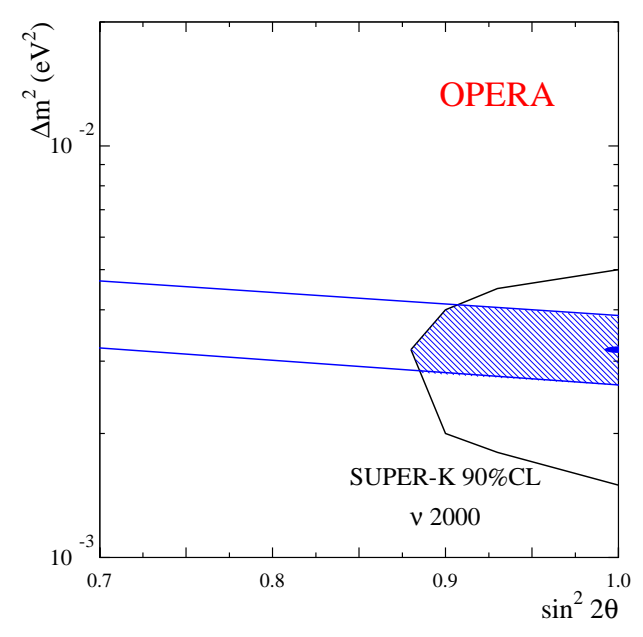

Figure 5: Possible 90\%C.L. allowed region for the oscillation parameters from OPERA after 5 years of running assuming an oscillation signal.

\section{References}

[1] A. De Santo, 'Int.

[2] Y. Fukuda et al., Phhys. Rev. Lett. 81 (1998) 1562 .

[3] Plenary talk at the XIX International Symposium on Lepton and Photon Interactions at High Energies, Stanford, 1999, hep-ex/9912007:

[4] K. Nakamura et al. 'Nucl. Phys. 91 (Proc. Suppl. 2 (2001) 203; S. Ahn, hep-ex/0103001, subm. to Phys. Rev. Lett..

[5] MINOS collaboration, The MINOS Detectors Technical Design Report, Fermilab, NuMI-L-337.

[6] For a description of the analysis see http:// ]www-numi.fnal.gov:8875/blessed/index.htmli

[7] G. Acquistapace et al., CERN 98-02, INFN/AE-98/05;

R. Bailey et al., CERN-SL/99-034(DI), INFN/AE-99/05.

[8] M. Guler et al., CERN/SPSC 2000-028, SPSC/P318, LNGS P25/2000.

[9] S. Aoki (for the OPERA Coll.), in Frontiers in Contemporary Physics 2001, Vanderbilt University, Nashville, Tennessee, USA (March 2001). 